Aus der geburtshülflieh-gynäkologisohen Klinik in Basel.

\title{
Beitrag zu dem Verhalten des Milchflusses bei Stillenden.
}

\author{
Von \\ Dr. Wilhelm Leube, \\ Volontärarzt.
}

(Mit 2 Abbildungen.)

Kehrer ${ }^{1}$ ) hat das Verdienst, zuerst genauere Untersuchungen über den freien Abfluss von Milch bei Stillenden ausgeführt zu haben. Er beschreibt eine Reflexerscheinung der Brüste, die er physiologischen Milchfluss nennt - ,theils ganz spontan, theils nach dem Anlegen des Kindes stellt sich ein Gefühl von Rieseln in beiden Brüsten und gleich danach ein von deutlichem Turgor der subcutanen Brustvenen begleiteter doppelseitiger Milchfluss ein" - . Dieser Fluss beginnt gewöhnlich nach den ersten 3-4, manchmal 8 Tagen nach der Geburt; er fehlt bei ca. 20 Proc. der Wöchnerinnen. „Ein sehr reichlicher Fluss ist Symptom starker Milchabsonderung, schwacher und kein Fluss kann jedoch auch bei reichlich secernirenden Frauen vorkommen." Als Minimum hat Kehrer 0,02, als Maximum 120,95 g, als Mittel für die rechte Brust 13,3, für die linke 16,56 g gefunden. Die Ausflussmenge beträgt im Mittel 8 Proc., im Maximum 16 Proc. des Gesammtinhaltes der betreffenden Brust.

Unter die Bezeichnung des physiologischen Milchflusses lassen sich nicht alle Beobachtungen von Milchabfliessen bei Stillenden bringen.

So publicirte Herff ${ }^{2}$ ) einen Fall, bei dem ,, trotz des reichlichen und beständigen Abflusses der Milch die Production der-

1) Beiträge zur vergleichenden und experimentellen Geburtskunde, Bd. I, Heft 4.

2) Beitrăge zur Lehre der Galaktorrhoe. 1889. Heuser'scher Verlag. 
selben sogar eine verminderte war"; es ging im Mittel 40,76 Proc., in den ersten 10 Beobachtungstagen durchschnittlich 64,45 Proc. der täglichen Gesammtmenge durch Abfliessen verloren.

Guénea u ${ }^{1}$ ), Scanzoni ${ }^{2}$ ), Schwarze ${ }^{3}$ ) berichten von Frauen, die durch hochgradigen Milchfluss in ihrem Allgemeinbefinden schwer gestört wurden.

Auf Anregung von Herrn Professor Fehling habe ich mich mit Messungen der ausfliessenden Milch bei Stillenden beschäftigt. Es sei mir gestattet, demselben für die gütige Ueberlassung des klinischen Materials, sowie für seine Unterstützung bei dieser Arbeit meinen aufrichtigsten Dank auszusprechen.

Meine Beobachtungen erstrecken sich auf 100 Stillende; die Beobachtungsdauer beträgt in der Regel 11-12 Tage, eine durch die hier übliche Entlassung der Wöchnerinnen bedingte Beschränkung.

Unter diesen 100 war

bei 63 Milchfluss vorhanden, " 37 fehlte derselbe.

A. Von den 63 , bei denen Milchfluss da war, waren

$$
\begin{aligned}
\text { Iparae } 31=49,2 & \text { Proc. } \\
\text { II- } " 18 & =28,6 \quad " \\
\text { Multi- } " \quad 14 & =22,2 \quad "
\end{aligned}
$$

B. Bei denen, wo er fehlte, lauten die entsprechenden Zahlen

$$
\begin{aligned}
\text { Iparae } 11 & =29,8 \text { Proc. } \\
\text { II- } " 5 & =13,5, \\
\text { Multi- } " 21 & =56,7 \quad,
\end{aligned}
$$

Die Entwicklung der Mamma war

$$
\begin{aligned}
\text { A. bei } 15 \text { schwach } & =23,9 \text { Proc. } \\
\Rightarrow 12 \text { mittel } & =19,0 \quad \\
" 36 \text { gut } & =57,1 \quad \\
\text { B. bei } 9 \text { schwach } & =24,3 \text { Proc. } \\
" 11 \text { mittel } & =29,8 \quad " \\
" 17 \text { gut } & =56,7 \quad "
\end{aligned}
$$

Bei A genügte zur Ernährung der Kinder bei $38=60,3$ Proc. die Muttermilch, bei 39,7 Proc. musste noch Kuhmilch gegeben werden.

1) Schmidt's Jahrbücher, Bd. XCI.

2) Lehrbuch der Geburtshülfe.

3) De Galaktorrhoea. Inaug. Diss. Gryphiae. 
Von den Kindern, die Muttermilch allein hatten, haben am 12. Tage

$$
\begin{aligned}
& \text { qugenommen } 16=42,1 \text { Proc. } \\
& \text { abgenommen } 22=57,9 \quad "
\end{aligned}
$$

Die entsprechenden Zahlen für $\mathrm{B}$ lauten

17 und 20, d. h. 45,9 gegen 54,1 Proc.

Zunabme der Kinder $12=70,6$ Proc.

Abnahme " " $\quad 5=29,4$,

Aus diesen Zahlen ergiebt sich ein starkes Vorwiegen der I- und II parae bei den Stillenden mit Milchfluss, der Mehrgebärenden bei denen ohne Fluss, während sonst in der Klinik das Ver-

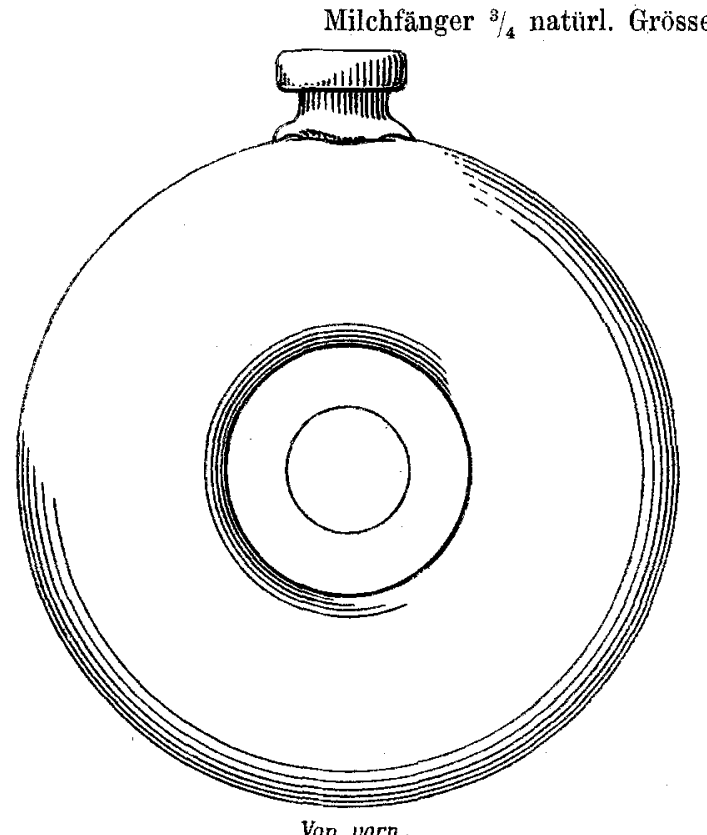

Von vorn.

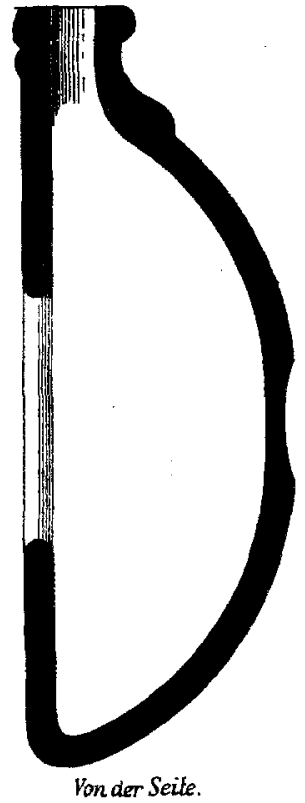

hältniss der Erstgebärenden zu den Mehrgebärenden wie $1: 1,6-2,0$ ist; ferner eine annähernde Gleichheit der procentischen Verhältnisse betreffs der Entwicklung beider Brüste; ebenso dass zwar procentisch bei Frauen mit Milchfluss etwas weniger oft Kuhmilch gegeben werden musste, als bei den anderen, aber die Kinder ersterer, die nur Muttermilch hatten, in bedeutend geringerer Zahl $(42,1$ Proc.) zugenommen haben, als die der letzteren (70,6 Proc.).

Der Durchschnitt der Kinder, welche am Entlassungstage sonst Gewichtszunahme zeigen, beträgt in den letzten Jahren 47 Proc. 
Es folgen die Aufzeichnungen von 25 Fällen, bei denen die einzelnen Ausflussmengen mit dem beigezeichneten Milchfänger (s. S. 12) - es gelang mir nicht, den Namen des Autors zu finden - aufgefangen und dann gemessen wurden; die Zahlen bedeuten Cubikcentimeter.

Bei den Milchmengen, die während des Stillens aus der freien Brust ausliefen, ist für diese angegeben, ob aus der rechten oder linken; für die Milchmengen, die in der Zwischenzeit gesammelt worden sind, ist dieser Unterschied nicht durchgeführt. Bei den Fällen, in denen die Milchmengen auch von der Nacht bestimmt sind, ist für die Nachtmenge Saug- und Spontanfluss nicht getrennt.

12. 8 Uhr Vm. $5 \quad 0 \quad-$ 10

13.

14. aufgehört.

Nr. II. Geburt 8. Juli.

12. 8 Uhr $\mathrm{Vm}, 5$

13. 10

$8 \quad " N "$ - $\quad 4,5-$

14.

$$
81 / 4
$$

8

4

$"$ V" 0 Vm. 5

$$
\mathrm{Nm} .8
$$$$
\text { - }
$$

$81 / 4$

1

$11 / 4$,

15.

6$$
\text { " }
$$$$
\mathrm{Vm}-13=
$$
$12^{1}$

16.

$6 " \Rightarrow \overline{11,2}-0$$$
5
$$$$
1
$$

$11 / 4$

17. 5

$\begin{array}{llllll}5 & \text { N } & - & 5 & - \\ 7 & \text { Vm. } 12 & - & - \\ 1 & \text { Nm. } 10 & - & -\end{array}$
Tag Stunde R. L. spontan 17. 8 Uhr Nm. - 8 18. ${ }^{12} 8^{1 / 2} "$ Vm. $15-15-$ $8 \frac{3}{4}$, - Tropfen

$21 / 2$
$2^{3 / 4}$

19. $4^{1} / 2 "$ Vm. 10 - $13,8-$

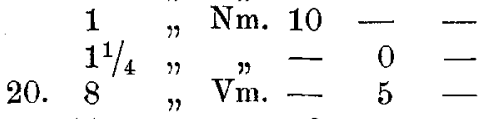
11

Nr. III. Geburt 10, Juli.

15. 12 Uhr M. $11-$

1 "Nm. - 0 -

5

16. 11

1

4

17. 5

(1)

V"m. 14

$\mathrm{Nm} .1$

"

政

Nm. 18

$1-4$

5

18. 8

19. 7

11

3

6

20. 7 
14 Leube, Beitrag zu dem Verhalten des Milchflusses bei Stillenden.

$\begin{array}{llll}\text { Tag } & \text { Stunde } & \text { R. } & \text { L. spontan } \\ 20.12 \text { Uhr M. } & - & 0\end{array}$ $121 / 4$ "Nm. $0-7$ -

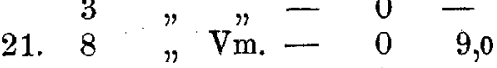
$11 ", \quad-0,5-$ $\begin{array}{ccccc}4 & \text { Nm. } & 0 & -\end{array}$

Nr. IV. Geburt 13. Juli.

16. 2 Uhr Nm. - 5 -

17. 8 " 8 " $15,5-2$ -

1 " Nm. - 0 -

$5 \quad " \quad " \quad-\quad \begin{aligned} 11 & - \\ 7 & \quad\end{aligned}$

18. 8 "Vm. 0 -

19. $7 " n-2$ -

$11 " n 0 \div-$

$3 \quad " N \mathrm{Nm} .-0$ -

$5 \quad " \quad 00-$

20.

$5^{1 / 2} " n-0$ -

$11 " \mathrm{Vm} . \quad 0 \quad-0$

1 "Nm. 0 - 2,0

$5 \quad " \quad 16-$

21. 8 "Vm. - 11 -

$11 " n-2,25$

12 "M. 0 -

3 " Nm. 0 -

$6 ", \quad-0$ -

$61 / 4 " \quad " \quad 0 \quad-\quad-$

22. 6 "Vm. - 0 -

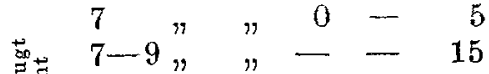

$9-10, \quad-11$

$10-10^{1 / 2} "$ - 25

11 (R. 17 L. 8)

2 Nm. 0 - $2,0-15$

$2 \frac{1}{2} "$ Nacht $"-\quad-\frac{15}{28}$

23. 7 Uhr Vm. $4-$

$8 " \quad$ - 5,5 -

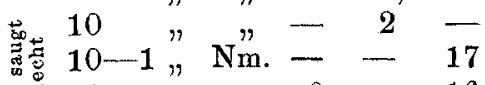

马귱 1 " 16

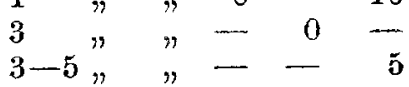

Tag Stunde R. L. spontan

23. 5 Uhr $\mathrm{Nm}$. 0 - -

Nacht - - 30,5

24. 6 Uhr Vm. $0-13,2$

$$
\begin{aligned}
& \begin{array}{l}
10 \\
10-2 \\
2
\end{array} \\
& 21 / 4 " \quad \text { " } " 0 \text { - } \\
& 3-5 " \quad "-3 \quad 17 \\
& 5 \text { 5-6" " } " \text { - } 21
\end{aligned}
$$

25. 6 (R. 14,8 L. 6,2)

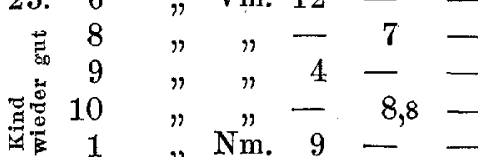

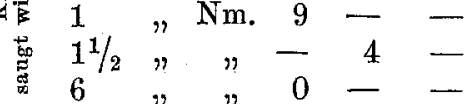

26. 8 "Vm. 10 -

$8^{1 / 4} "$ M" $\overline{25}-$

$12^{1 / 2} " \mathrm{Nm} .-11-$

3 " $" 23-$

$3 \frac{1}{4} " \quad " \quad-20$ -

6 " $" 11-$

$6^{1} / 4 ",-3-$

27. 6 " $" \mathrm{~mm} .6-$

$6^{1 / 2} " n-5-$

$\begin{array}{rrrrrr}8 & \prime & \prime & 3 & - & -\end{array}$

Nr. V. Geburt 15. Juli.

24. 6 Uhr Nm. - $3,2-$

25. 2 "Vm. 2 -

$11 " \quad " \quad \overline{0}_{6} 13,2-$

2 "Nm. - 12,3 -

$31 / 2 \quad " \quad$ " $1,1-$

26. $\begin{array}{llll}7 & 7 & & \\ 7 & & 1,2 & - \\ 7 & \end{array}$

27. $4^{1 / 2} " \quad$ - 10 -

$81 \% 2 " \quad$ - $1,5-$

Nr. VI. Geburt 16. Juli.

19. $7 \mathrm{Uhr} \mathrm{Vm}$. Tropfen -

20. $81 / 2 ", \quad$ - 0,4 -

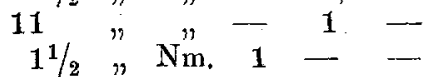


Leube, Beitrag zu dem Verhalten des Milchflusses bei Stillenden.

\begin{tabular}{ccccccc} 
Tag & \multicolumn{3}{c}{ Stunde } & R. & \multicolumn{2}{c}{ L. spontan } \\
20. & 3 & Uhr Nm. & - & 1,2 & - \\
6 & $n$ & $\#$ & 3,0 & - & - \\
8 & $n$ & & - & 1 & -
\end{tabular}

21. Kind trinkt nicht mehr. Pneumonie Exitus lethal. Kein Spontanfluss.

Nr. VII. Geburt 16. Juli. 18. Tropfen 18. -24 . (1) 24. $9 " n-2,0-$ 25. 5 "Vm. 15,0- $71 / 2 " \quad$ " $2,2-$ $\begin{array}{rlll}9 & \text { " } & 2,3- & - \\ 12 & \text { M. } & 0 & -\end{array}$ 4 "Nm. - 13 26. 7 "Vm. 4 $11 ", \quad-0$ $4 \quad$ Nm. - $16,0-$ 27. $\begin{array}{llllll}5 & \text { " } & 1,1- & -\end{array}$ $\begin{array}{rrrrr}11 & \Rightarrow & 0 & - & -\end{array}$ aufgehört.

Nr. VIII. Geburt 17. Juli.

19. 9 Uhr Vm. - $14-$

$11, "$ - 8 -

3 "Nm. $10-$ -

$\begin{array}{llllll}5 & n & n & - & 9 & -\end{array}$

$8 \% " \quad 10-$

20. 8 "Vm. 14 - -

$10 \quad " \quad$ - 4 -

$11 ", \quad-9,4-$

$1 \quad " \mathrm{Nm} .6-$

$5 \quad " n-16,6-$

$6 "$ " $\quad 1,6-$

21. 9 "Vm. $15-$

$10 \quad n \quad n \quad 5,5-$

$1 \quad " \quad 3 \quad 3 \quad-$

$21 / 2 " n=0$ -

$4^{1 / 2} ", \quad " 5-$

22. 8

$$
\begin{aligned}
& 8 \% \text { " } 12 \quad-\quad- \\
& 1 \quad \text { Nm. }-7 \quad 7 \quad 4,6 \\
& \begin{array}{llllll}
2 & \prime & \prime & 4 & - & 3 \\
5 & \# & 1 & - & 2 & 4
\end{array}
\end{aligned}
$$

Tag Stunde R. L. spontan

23. 8 Uhr Vm. - 1,4 -

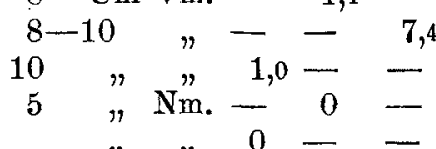

aufgehört.

Nr. IX. Geburt 24. Juli.

25. 8. Uhr Vm. 'Tropfen -

$10 " \Rightarrow-1,23$

$1 \quad$ Nm. - 2,2 -

26. 10 V.m. 2 - -

12

4 " Nm. - 0 -

27. 8 " $8 \mathrm{~m} .2$ - 2

$11 " \quad \cdots \quad--17,3 \quad 14$

$1 \% \mathrm{Nm} .10-15$

$4 \quad " \quad-9 \quad 9-$

$6 " n \quad 2,5-10$

28. $9-9 "$ Vm.

9 " $\quad$ - 5 -

$10 \quad " \quad " \quad 5 \quad-\quad 61$

2 "Nm. $4-25$

$4 \quad " \quad n \quad-\quad 8 \quad-$

$5 \quad " \quad 2 \quad 2 \quad-$

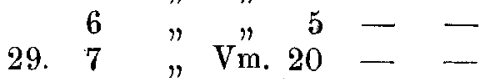

8 " $"$ " 343

1 "Nm. - 11 -

$\begin{array}{llllll}3 & " & 11 & - & - \\ 6 & " & & 35 & -\end{array}$

30. 8 "Vm. - 34 -

$10 \quad " \quad$ " $15 \quad-21$

$11 \frac{1}{2} \quad, \quad, \quad-\cdots \quad 0$

$2 "$ Nm. $0-20$

$\begin{array}{rrrrr} & 4 & & & \\ 31 . & 7 & & 0 & 8,2\end{array}$

$8 " n=6$ - $" 6$

$\begin{array}{r}11 \\ 2\end{array} \quad \mathrm{Nm}-0$ -

1. $5 "$ " $" 8=$

1. 8 " Vm. $0-$

1. " Nm. 3,2

$\begin{array}{llllll}2 & \prime & - & 1,3-\end{array}$

2. $7 \quad$ 7 vm. $\begin{array}{rrrr}15 & - & -\end{array}$ 
I6 Leube, Beitrag zu dẹ Verhalten des Milchflusses bei Stillenden.

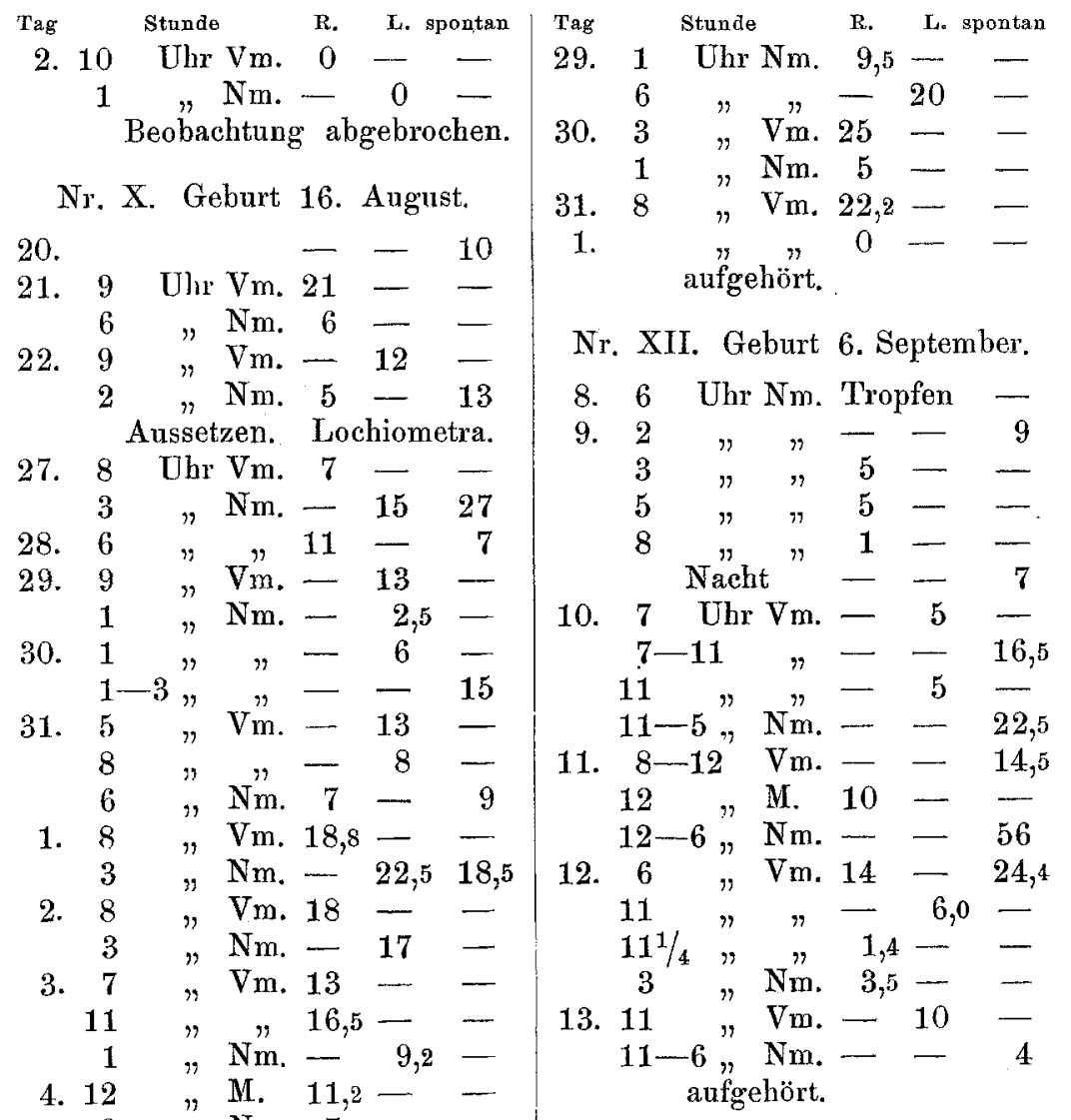

Nr. XIII. Geburt 7. September.

9. 8 Uhr Nm. - $14,5-$

5. 5 "Vm. $12,5-$

10. 6 "Vm. - $3,0-$

$10 " \quad,-29,2-$

$3 \%$ Nm. 10,5 - -

$\begin{array}{llllll}7 & \prime & n & 24 & - & -\end{array}$

Nr. XI. Geburt 20. August.

27. 1 Uhr Nm. $10 \quad \overline{21}-$

28. $\begin{array}{rllll}31 & \text { "Vm. } & - & 21 & 6 \\ 11 & -\end{array}$

11-1" Nm. - 20

$1 \quad " \quad 11-$

$3 \quad " \quad-7$ -

29. $\begin{array}{llllll}5 & 9 & & 15 & - & - \\ & & & \end{array}$

$11 " n-19 \quad 17$

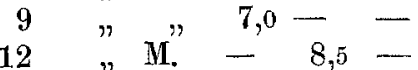

2 "Nm. 10,0 - -

$3 "$ " $" 14,5-$

$4 ", \quad-11,2-$

$4^{1 / 4} " \quad " \quad 5,0-16$

11. 6 " $\quad \mathrm{Vm}$. 7,0 -

12. $6 " n-16,072$

$8 " n 7,5-8,8$

$11^{1 / 2} \quad " \quad, \quad-3,4 \quad 11$

3 "Nm. 4,0

$5 \quad "$ " $\quad 6,89$

13. 7 " Vm. - 6 " $6,9=$

$8-11 " \quad 4 \quad 4,4-\overline{33,6}$ 
Leube, Beitrag za dem Verhalten des Milchflusses bei Stillenden.

Tag

13. 11 Uhr Vm. - $10,0-$ $11-3, \mathrm{Nm} .-241,2$ $3 \quad " \quad 3,5-\quad-$ 14. $8-10 \mathrm{Vm} .-26,3$ $10 " n-6,2-$ 13 " M. $4,3-$ $12-4 " \mathrm{Nm} .-48,5$ $4 " \quad " \quad-3,5-$ $4-6 ", \quad-25,5$ Nachts - - 29,4

15. 6 Uhr Vm. - $5,4-$ $6-11 \quad "-\quad 22,9$ 11 Uhr " $8,8-$ $11 \frac{1 / 4}{11} " \quad-0,4-$ $11^{1 / 2}$ " "elektr. - $111 / 2-3 \mathrm{Nm} .-25,2$ 3 Uhr $"-0,2-$ $5 \quad "$ " elektr.- $5^{1 / 2} "$ Nachts" $\quad$ - 28,4

16. 6 Uhr Vm. 6,2- -

$10 \quad " n-5,1-$

$3 \quad " N m .8,5-$ -

$5^{1 / 2} \quad " \quad " \quad 2,0-\quad-$

$6 " \Rightarrow-1,6-$

17. 6 Uhr Vm. $10,5-25,0$

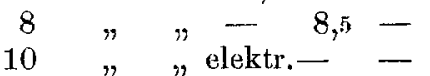
10-1" Nm. - - 6,8 $1 \quad " \quad$ " elektr. $4,9-$

$6 "$ " $" 0$ -

18. $8 \underset{\text { Uhr Vm. }}{\text { Nachts }}-7,5-$

Nr. XIV. Geburt 14. September.

18. Abends Tropfen - -

19. 12 Uhr M. $10,2-10,4$ $20 . \quad-\quad 0 \quad 2,2$ $21 . \quad 0-15,5$ 22. 11 Nachts $\quad-\quad-41,2$ 23. aufgehört.

Archiv f. Gynäkologie. Ba. XLIII, Hft. 1.
Tag Stunde R. L. spontan

Nr. XV. Geburt 18. September.

22. 7 Uhr Vm. 21,8 -- -

9 " $" 7,2-$

$9-2 " \mathrm{Nm} .-28,8$

$2 \quad " \quad 6,8-6$

$41 / 2 \quad " \quad " \quad-1,6 \quad-$

$6 \quad " \quad 9,2-46,6$

23. $7 \quad " \quad V m \cdot 14,8-\quad 2,0$

$11 " \#-5,2-$

$2 \quad " N m .-1,2 \quad 14,0$

$5 \quad " \quad-0,2-$

24. $6 \quad$ "Vm. - $1,6-$

$8 \quad " \quad, 9,5--$

$9 \quad " \quad 3,2--$

$9-1, \mathrm{Nm},--47,2$

$1, " \quad$ - $8,5-$

$41 / 2 "$ " Compress. der linken Warze bei Beginn des 'Trinkens 8,8 -

5-7 Uhr Nm. - $\quad 4,4$

25. 7 "Vm. $-13,2-$

$81 / 2 \quad " \quad 7,5-21,8$

$11 " \Rightarrow 2,0-25,0$

$1 \frac{1}{2} \Rightarrow \mathrm{Nm} \cdot 17,5 \ldots$

$2-5, " \quad-\quad-48,5$

$5 \quad " \quad-13,2-$

26. 6 "Vm. - 0 -

$6-8 " \quad " \quad-21$

$8-11 " \quad-34,2$

$11,10 " \quad$ elektr.- -

$11 " \Rightarrow-$ - 10,0

-12 "M. - - 7,2

$12-3, \mathrm{Nm} .-\quad \mathrm{9}, 8$

$3-3 / 4, "-\ldots 12,8$

$3 / 45$ Uhr $",-0,6-$

$5 \%$ " elektr.- -

$51 / 2-7 "$ " - - 15,6

$8 \mathrm{Uhr} "-5,0-$

27. $71 / 2-9 \mathrm{Vm}$. $-19,8$

$\begin{array}{rcccc}8 & \text { Uhr } & 0 & 0 & - \\ 9 & " & \text { Antipyrin } & 0,25 \\ 11 & " & " & " & 12,4 \\ 1 & " & \text { Nm. } & " & 0 \\ 3 & " & " & " & 0 \\ 5 & " & " & n & 0 \\ 7 & n & n & n & 0\end{array}$ 
18 Leube, Beitrag zu dem Verhalten des Milchflusses bei Stillenden.

Tag Stunde R. L. spontan

28. im Laufe des Tages 46,4 29. $8 \frac{1}{2}$ Uhr Vm. 0,25 Antip. $-10 " \# \quad-\frac{0,25}{10}$ Antip. -12 "M. - -0

Nr. XVI. Geburt 21. September. 23. 6 Uhr Nm. - 14,5 24. 8 Vm. - $10,0-$ 4 "Nm. $30,8-23,0$

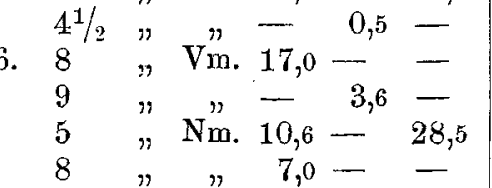
26 28. 8 "Vm. - - 38,2 $11 " \quad " 3,5-\quad$ 1 "Nm. - $6,0-$ $3 \quad " \quad 10-1$ $8 \quad " \quad, 15-$ $11 " n-7,5-$

29. 7 "Vm. $8,4-$

$11 " \quad$ verschüttet -

1 "Nm. 11,0 - -

$3 ", \quad-25,0$

30.

6 $5 \quad$ Vm. 4,8- -

$2 ., \mathrm{Nm} .-16,5-$

$3 \quad " \quad, 15,5-$ -

$5 \quad " \quad " 30,2-$

1.X. $6 " n$ Vm. $6,0-7,5-$

8 1.X. $6,0-3,-$

8 " N" $\overline{47,0}-3,5-$

$3 \%,-23$ -

$6 \% "$ - 17 -

8 " " 25,5- -

$12 " \prime-30-$

2. 5 " Vm. 15,5- -

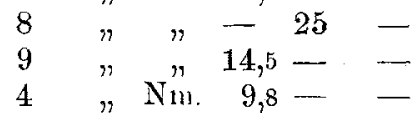

Nr. XVII. Geburt 22. September. 24. 5 Uhr Nm. 'Tropfen 25.

$\begin{array}{lllll}26 . & 12 & \text { M. } & 2,2- & - \\ 51 / 2 & " N & \text { Nm. } & -\end{array}$
Tag Stunde R. L. spontan

27. 7 Uhr Vm. - 24 -

$11 " \quad 3-9$

8 "Nm. - 9 -

28. 9 "Vm. $25-$

4 "Nm. - 16,2 -

$61 / 2 " n-13$ -

29. $\begin{array}{cccccc}9 & 8 & & 5 & - & -\end{array}$

$9 \quad " \quad 3,8-\quad-$

$2 \quad$ "Nm. - $12 \quad 2,0$

$4 \frac{1}{2} ", \quad-23,6$ -

30. 8 "Vm. $18-$ -

$11 ", \quad 3,8 \mathrm{ma}-$ nuelle Compr. d. Warze

6 Uhr Nm. - $17 \quad 19$

$6^{1 / 2} "$ " $1,4-$ -

1. X. $81 / 2 "$ Vm. $43,6-$ $81 / 2-11,-4,8$

11 Uhr " elektr:- $11-3, \mathrm{Nm}$. - $\quad 5,8$

$5 \quad " \quad, 15,2-\quad$

2. 7 "Vm. $\quad$ - 13,0 -

$10^{1 / 2} \quad " \quad, 12,8-\ldots$

$11 " n$ elektr.- -

2 "Nm. - 22,6 -

$4 " \Rightarrow 6,2-0$

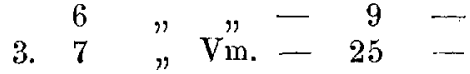

10

2

5

4. 6 " v" $\overline{15,5} 4$ -

4. 6 " 9 V $15.15,5-18$ -

12 "M. - 27,5 -

Nr. XVIII. Geburt 1. October.

3. 11 Uhr Vm. - 24,5 -

1 "Nm. 14,5 - -

4. 10 "Vm. - $\begin{array}{rrr}10,0 & \\ 13 & \end{array}$

1 Nm. $15,5-$

$5 \quad " \quad-10,0-$

$6 " \Rightarrow 33,5-\ldots$

5. $8 \quad " V \mathrm{Vm} .-3,0-$

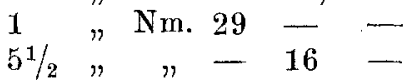


Leube, Beitrag zu dem Verhalten des Milchflusses bei Stillenden.

\begin{tabular}{|c|c|c|c|c|c|c|}
\hline ag & & Stunde & & R. & L. 8 & pontar \\
\hline 6. & 8 & Uhr & Vm. & 17,2 & - & \\
\hline & 2 & $\eta$ & $\mathrm{Nm}$. & - & 26 & \\
\hline & 5 & " & $n$ & 6,2 & - & \\
\hline & 8 & $"$ & $"$ & - & 14,2 & \\
\hline 7. & 9 & $"$ & $V \mathrm{~m}$. & $\overline{7}$ & 15,4 & $=$ \\
\hline & $\begin{array}{l}1 \\
5^{1} / 2\end{array}$ & $"$ & $\mathrm{Nm}$. & & $\overline{3,8}$ & - \\
\hline
\end{tabular}

8. 8

10 " Manuelle Compress. der linken Warze, sofort nach Aufhören des Stillens

$$
10,0-
$$

1 Uhr Nm. dito rechts $1-4, \quad " 24,0$ - $5 "$ "Digitalcompression, links Auslaufen einiger Tropfen während derselben, sofort nacher - 9,8 -

$5-8 \mathrm{UhrNm} .-\ldots 15,0$

9. 12 M. $-24,5-$

1 "Nm. - 8 -

$\begin{array}{lllll}3 & \quad & \quad & 6,0- & - \\ 6 & & -\end{array}$

10. 7 "Vm. - 10,0 -

11 " "Dig.-Compress. rechts, in den nächsten $10 \mathrm{Min}$.

12-4 Uhr Nm. $-\frac{1,2-\overline{49,5}}{-}$

11. 9 "Vm. $10-$

$11 ",-34$ -

2 "Nm. 5 -

$4 " n-28$

7. " " 39 -

2 "Nachts - 44 -

12. 6 "Vm. $4,5-$

$\begin{array}{rlllll}9 & \prime & \prime & - & 25 & 5,5 \\ 11 & \prime & \prime \prime & 4,5 & -\end{array}$

Nr. XIX. Geburt 4. October.

6. Mittags - $-56,2$ 7 Uhr Nm. 12,8 -- -

7. $81 / 2$ Vm. $33,6-16,4$ $2 \% \mathrm{Nm}$. - 26,6 $2-5 \% \quad \%-\quad-45,4$ $5-7 " \quad " \quad 25,2-\overline{12,6}$ Nachts - - 30,4

8. 9 Uhr Vm. $39,2 \ldots-$
Tag Stunde R. L. spontan

8. 2 Uhr Nm. $51,0-48,2$

9. 8 " " verschüttet -

9. $8 \quad "$ Vm. $1,8-\ldots$

$11 " \quad " \quad-4,0-2$,

$3 \quad$ Nm. $4,6-\ldots$

10. 8 " 8 Vm. - $\begin{array}{r}0,8 \\ 27,2\end{array}$

$11 / 2 " \mathrm{Nm} .9,0-19$

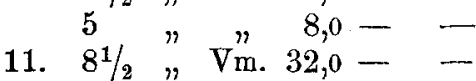

$11^{1 / 2} " 2 n-9,6$

$4 " n 7,2-$

12. 6 " $9 "$ - $18,0-$

12. 9 "Vm. - $9-$

$2 \quad " \mathrm{Nm} \cdot-\frac{5}{1,2}-$

13. 8 "Vm.12,0- -

$11 "$ " $" 0$ -

14. $" \mathrm{Nm} \cdot \frac{-}{0}-10,0=$ aufgehört.

Nr. XX. Geburt 8. October.

10. $2^{1} / 2$ Uhr Nm. - $3,8-$

11. $81 / 2 "$ Vm. $\quad$ - $2,6-34,4=$

4 " Nm. 24,0 - 41,8

8 Nachts" - $17,2-$

12. $81 / 2$ Uhr Vm. - $17,0-$

2 "Nm. 22,6 - 72,4 , $4^{3 / 4} "$ "Compression der linken Warze vom Beginn des Stillens, bis 10 Minuten nachher

$$
\text { 5-6 UhrNm. - } 12,2-
$$

13. 8 "Vm. 2,4 97,6

1 "Nm. 19,2 - -

$5 \% \ldots-57,8-$

$5-6 " \Rightarrow \quad " \quad-25,0$

14. 8 "Vm. - $2,4-$

2 "Nm. - 4,2 28,2

15. 8-12 M. - - 37,8

16. 2 "Nm. - 32,0 -

16. 8 "Vm. 25;0 11

17.

aufgehört. 
20 Leube, Beitrag zu dem Verhalten des Milchflusses bei Stillenden.

$\begin{array}{ccc}\text { Tag } & \text { Stunde } & \text { L. spontan } \\ \text { Nr. XXI. Geburt 16. October. }\end{array}$

20. Abends - $-10,5$ Nachts - - 34,5

21. 9 Uhr Vm. - $14,5 \quad 7,2$

22. R. abgesetzt, spontan R. 4,0.

Nr. XXII. Geburt 14. October.

20. Abends - $\quad 6$

21. $\quad-\quad-16,5$

22. 10 Uhr Vm. $9,5-\quad-$

12 M. $-11,5-$

$2 \quad " N \mathrm{Nm} . \quad 7 \quad 7,5$

23. $11 "$ Vm. $12-$ -

$2 \% \mathrm{Nm}, 9,5-$

$6 \quad, \quad 6--$

24. $10 " \mathrm{Vm} .5-7,0$

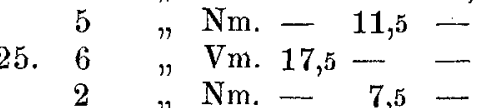

26. 6 " 6 Vm. $10,0-$ -

$6-9 " \mathrm{Nm}$. - $-5,0$

Nr. XXIII, Geburt 1. November.

5. 4 Uhr Nm. - $17,5 \quad 6,5$

$6 "$ " $14,5-$

6. 7 "Vm. -6 -

12 M. - 23,5 -

$2 \quad " \mathrm{Nm} .4,0--$

$5 "$ " $" 15-$

$9 \quad " \quad 15 \quad-$

$\begin{array}{lllll}7 . & 6 & \text { "Vm. } & -23 & 9\end{array}$

$8 \quad " \quad-22-$

$11 " \# 12,5-7,5$

$2 \quad " \mathrm{Nm} .-14,5-$

3 " "elektr. - -

$4 " \# 0$ -

$8 "$ " verloren -

8. 9 "Vm. $11-$

$11 " n-20-$

$41 / 2, N$ Nm. elektr. -

$5 " n-20$ -

9. 7 " 7 Vm. $\frac{25}{10}-$

12 M. -2012

$3 \quad \mathrm{Nm} .30-$

Fluss bis zum Austritt.
Tag Stunde R. L. spontan

Nr. XXIV. Geburt 12. November. 14./15. Nachts Menge verloren.

15. 11 Uhr Vm. - 6,5 -

$1 " N \mathrm{~m}, 28-8$

16. 8 "Vm. $26-4$

$11 " \Rightarrow-10-$

$11-6 " \mathrm{Nm} .-\quad-57,5$

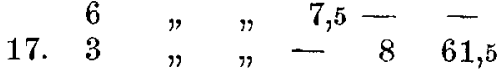

$5 \quad " \quad-3-$

Nachts - $\quad 19$

18. 6 Uhr Vm. - $2-$

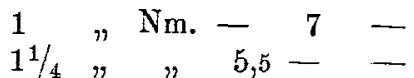

$4 \frac{1}{1 / 2} " \quad " \quad 3,5--$

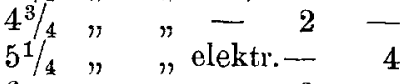

6 " $"-3,5-$

Nachts - $\quad 15$

19. 9 Uhr Vm. - 2,5

12 "M. 25,5 - -

3 "Nm. - 20,2 z. Th.

6 " verschüttet -

Nachts $\quad-\quad 5,5$

20. 6 Uhr Vm. 4,5- -

$\begin{array}{rrrrrr}8 & \prime & \prime & - & 3 & - \\ 10 & \prime & \prime & 3,5 & - & 14,5\end{array}$

12 "M. - 10,0 -

$4 " \mathrm{Nm} .2$ -

Nachts - - 6

21. 8 Uhr Vm. - $2,8-$

$2 \quad$ Nm. - $4,5-$

$4 \quad " 3-10$

$5 \quad " \quad$ " 15 -

$6 \% "-9-$

$8 \quad, \quad " 12,5--$

$81 / 4 " \Rightarrow \quad 6,5-$

Nachts - - 15

22. 8 Uhr Vm. $3,5 \div-$ 10

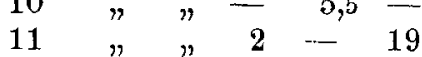

$3 " \mathrm{Nm} .6,5-2$

$6 " \quad$ " $4,5--$

23. 6 " "vm,

$8 \quad " \quad " \quad-3,5-7=$ 
Leube, Beitrag zu dem Verhalten des Milchflusses bei Stillenden.

Tag Stunde R. L. spontan

23. 11 Uhr Vm. $18-$

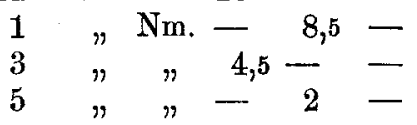

Nr. XXV. Geburt 12. November.

15./16. Ausfluss nicht gemessen.

17. 8 Uhr Vm. - $24-$

8-1, Nm. - - 77

$1 "$ " $322-$

$3-6 " \quad " \quad-\quad-61$

$6 \quad " \quad$ " $\quad 9 \quad-$

Nachts $\quad-\quad 254$

18. 6 Uhr Vm. $15-$

$6-1, \mathrm{Nm} . \quad-\quad 127$

$1 " \Rightarrow-27,5-$

$1-4 " \Rightarrow-50$

$4 \quad " \quad 23,5--$

5 5-6" " "elektr. - -

(R. 19,0 L. 15,0 spontan)

$6-10 " \#-24,5-\overline{28,5}$

$10 " \Rightarrow-25,5-$

Nachts - -65

19. 6 Uhr Vm. 34,5- -

$6-8, " \quad-20$

$8 " n-35-$

$91 / 2 " \prime \quad-24,5-$

$10-12 \quad " \quad-22$

12 M. 15 -

$2 \%$ Nm. 30,5 - -

$4 \quad " \quad-46,0 \mathrm{z} . \mathrm{Th}$.

5 " " elektr. -

$5-6 ", \quad$ - $\quad 23,8$
Tag Stunde R. L. spontan

19. 7-9 Uhr Nm. - $-47,5$

Nachts - $\quad-93$

20. 6 Uhr Vm. $12-$

$7-11 " \Rightarrow-10,5-$

$11 \%, 28-$

4 "Nm. - 18,5 -

$41 / 4 ", \quad 15-$

$4-7 ", \quad$ - $\quad 25,5$

$7 " \Rightarrow-12,5-$

$8 " \Rightarrow 15,5--$

Nachts - - 65

21. 6 Uhr Vm. $26,5-\quad-$

$8 \quad " \quad-24,5-$

$8-10 " \quad$ - $\quad 54$

$10 " 23,0-\quad-$

$10-2 " \mathrm{Nm}$. - $-15,5$

$2 " \quad$ - 14,5 -

$4 \quad " \quad " 24,5-$ -

5 5 6 " "elektr. -

$6 " \quad " \quad-\overline{12,5}-$

$6-8 " \quad,-\quad-49,5$

Nachts 25,5 46,5 -

22. 6 Uhr Vm. - 25 -

$7 " \quad 22 \quad-$

$7-11 " \quad-\quad-12,5$

11-3" N". 13 -

$3 \quad " \quad-16,5-$

$5 \quad " \quad 27-$

$5-7 ", \quad-\quad 16$

Nachts - - 48,5

23. 6 Uhr Vm. $34-$

$6-8, \quad, \quad-32,5$

8 " $"-24-$

$8-11 " \quad-8,5=10,5$ 
22 Leube, Beitrag zu dem Verhalten des Mïlchflusses bei Stillenden

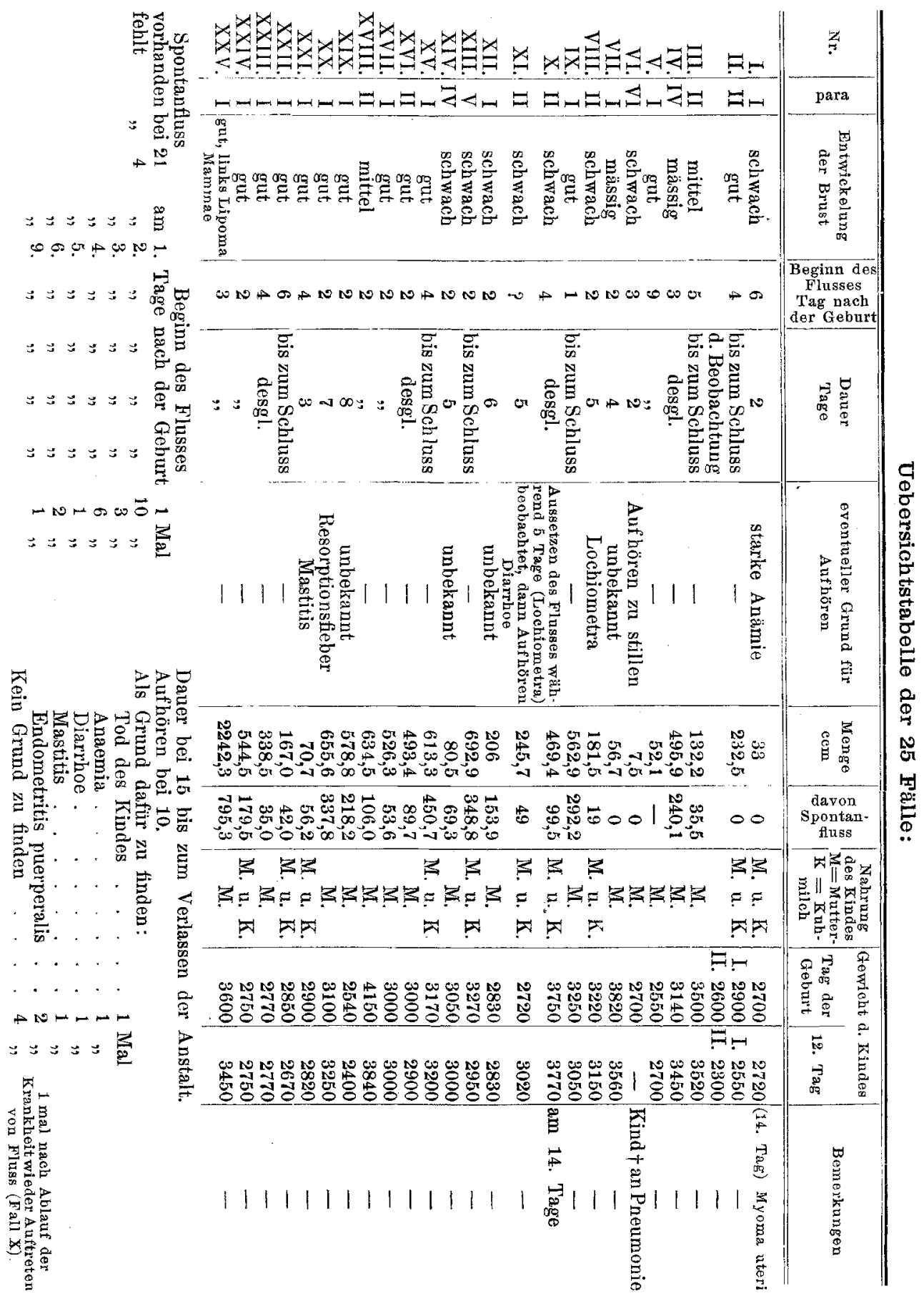


Vergleiche ich meine erhaltenen Resultate mit denen Kehrer's und Herff's, so kann ich die Angabe Kehrer's bestätigen, dass bei der Mehrzahl der Stillenden (63 Proc., Kehrer ca. 80 Proc.) sowohl nach dem Anlegen des Kindes ein Milchfluss aus der freien Warze erfolgt, als auch neben dem Saugflusse ein Spontanfluss vorkommt.

Kehrer sieht ,als das ausschliesslich in Betracht kommende Erregungsmittel des Milchflusses" das Saugen an. Der Saugfluss lieferte auch mir in der Mehrzahl der Fälle den grösseren Theil der abgeflossenen Milch. Doch betrug im 1. Falle (XIV.) die spontan ausgelaufene Milch über $3 / 4$, in 5 Fällen über die Hälfte (IX. XII. XIII. XV. XX.), in 3 Fällen (IV. XIX. XXV.) über ein Drittel der Gesammtmenge. Er fehlte nur in 4 Fällen.

Durch diese Zahlen dürfte der Spontanfluss eine grössere Bedeutung erhalten, als Kehrer sie ihm zuweist.

Dieser Fluss tritt, wie es auch Herff in seinem Falle beobachtet hat, bälder oder später nach Beendigung des Stillens zuerst meist aus der vorher zum Stillen nicht benutzten Brust, später aus beiden Brüsten zugleich ein; einen Grund können die Frauen gewöhnlich nicht angeben; das Gefühl des Einschiessens haben die wenigsten. Die Dauer ist verschieden, oft absatzweise, bei einzelnen ein fast continuirliches Abträufeln. Ein Vorkommen von Spontanfluss allein habe ich nicht gesehen, Saugfluss war immer daneben.

Ein Bild von Abhängigkeit des einen vom anderen giebt Fall.IV. In den ersten Tagen des aufgetretenen Flusses ist derselbe nur ein sich in geringen Grenzen haltender Saugfluss. Am 6., 7., 8., 9. Tage saugt das Kind schlecht, der Saugfluss hört fast ganz auf, dagegen läuft Milch spontan in reichlicher Menge ab, vom 10. Tage saugt das Kind wieder kräftig, der Spontanfluss geht zurück, der Saugfluss steigt bedeutend.

Dass die Kraft des Saugens Einfluss auf Eintritt und Stärke des Flusses hat, ist von Kehrer durch mehrere Versuche bewiesen.

Was den Beginn des Flusses betrifft, so ist nach meinen Zahlen der 2. Tag nach der Geburt der häufigste, ihm folgen der 4., 3., 6., 1., 5., 9. Kehrer findet, dass die ersten 3-4 Tage, manchmal selbst 8 Tage gewöhnlich ohne Fluss vorübergehen. Bezüglich der Dauer lassen sich meine Zahlen mit den Kehrer'schen nicht vergleichen, da meine Beobachtungen sich in der Norm nur auf 11 Tage erstrecken. 15 mal bestand der Milchfluss bis zum Ende der Beobachtung, 10 mal hatte er vorher aufgehört. 
Als Ursache für Aufhören des Flusses kennt Kehrer fieberhafte Puerperalprocesse, Mastitiden, ferner Eintritt der Menstruation und erwähnt als möglich Gemüthsbewegungen und mangelhafte Ernährung.

Fieberhafte Puerperalprocesse habe ich in 3 Fällen notirt; dass die Krankheit die Schuld an dem Aufhören des Flusses war, beweist Fall X., in dem nach Ablauf der Erkrankung der Milchfluss wieder eintrat.

Ausser Mastitis musste ich einmal das Auftreten von Diarrhoe, durch den von ihr bedingten Säfteverlust erklärlich, einmal eine hochgradige Anämie, bedingt durch Myoma uteri mit vorhergegangenen starken Blutungen, als Grund annehmen. In den 4 Fällen, wo keine Erkrankung nachzuweisen war, haben auch Gemüthsbewegungen nicht beobachtet werden können.

Die Ausflussmenge beträgt nach Kehrer im Mittel 8 Proc., als Maximum 16 Proc. des Gesammtinhaltes der Brust.

Nach den Untersuchungen von $\mathrm{Kr} \ddot{\mathrm{u} g e} \mathrm{r}^{1}$ ) beträgt die Menge der Milch, die in den ersten 11 Tagen rom Kinde getrunken wird, im Mittel $4313 \mathrm{~g}$, nach Hähner $\left.{ }^{2}\right) 3980 \mathrm{~g}$.

Das specifische Gewicht der Milch zu 1030 gerechnet, geben diese Zahlen in Cubikcentimeter 4187 resp. 3844. Lege ich nun 4000 meiner Rechnung ${ }^{3}$ ) zu Grunde - eigene Wägungen der Kinder fehlen mir -, so würde sich für die 8 Proc. Kehrer's eine Menge von $320 \mathrm{ccm}$, für die 16 Proc. $640 \mathrm{ccm}$ ergeben. Die Zahl 320 wird von 16, 640 von 2 Fällen bezüglich der aufgefangenen Milchmenge überschritten.

Wincke $1^{4}$ ) berechnet für die ersten 11 Tage ca. 5 l, von denen 25 Proc. verloren gehen $=1225 \mathrm{cbm}$. Hinter dieser Winckel'schen Zahl bleiben 24 meiner Fälle zurück, in einem ist sie noch fast um das Doppelte übertroffen. In letzterem Falle sind nach den erst angenommenen Zahlen 56, nach den zweiten 44,8 Proc. verloren gegangen, Zahlen, die den von Herff beobachteten sehr ähnlich kommen.

1) Dieses Archiv, Bd, VII.

2) Jahrbuch für Kinderheilkunde. Neue Folge. Bd. XV. 1880.

3) Die Zahlen von Krüger und Hähner sind für den Vergleich etwas zu klein, da bei diesen die abgeflossene Milch nicht ersichtlich.

4) Pathologie und Therapie des Wochenbettes. 
Die gesammelte Menge beträgt in 9 Fällen unter 8 , in 14 zwischen 8 und 16 Proc., in je 1 Fall 18 und 56 Proc. der vom Kinde getrunkenen Menge, dieselbe wie oben zu $4000 \mathrm{cbm}$ gerechnet.

Kehrer hält einen sehr reichlichen Fluss für ein Symptom starker Milchabsonderung.

Machen wir für diese Angabe die Probe durch Vergleich der Gewichte der Kinder am Tage der Geburt und am Morgen des 12. Tages, so müssten wir erwarten, dass die Kinder der Frauen mit Milchfluss, besonders die mit reichlichem, am Tage ihrer Entlassung das Anfangsgewicht überschritten, zum mindesten wieder erreicht haben. Denn nach Winckel ${ }^{1}$ ), Krüger ${ }^{2}$ ) u. A. nehmen die an der Mutterbrust in den ersten Tagen an Gewicht ab, haben meist vor dem 10. Tage den Gewichtsverlust gedeckt.

Von den 38 Kindern der Mütter mit Milchfluss, bei denen die Muttermilch genügte, haben 42,1 Proc. zu-, 57,9 Proc. abgenommen, während bei den Frauen ohne Milchfluss, die der eben genannten Bedingung entsprechen, die Zunahme 70,6 Proc., Abnahme 29,4 Proc. beträgt. - Von 12 Kindern, deren Mütter über $400 \mathrm{ccm}$. durch freien Abfluss verloren, haben 25 Proc. zugenommen, 25 Proc. sind gleichgeblieben, 50 Proc. haben abgenommen.

Diese Zahlen werfen ein eigenthümliches Licht auf den Reichthum an Milch bei Frauen mit Milchfluss. Was ist nun Schuld für diese Vergeudung der Milch?

Kehrer hält für die Ursache des Milchflusses die hyperämische Verdrängung der Milch. „Wenn die Drüsengefässe sich erweitern, so muss dadurch bei der rekativen Starrheit des Drüsengewebes ein Druck auf die Milchgänge ausgeübt werden, der bei relativ leeren Gängen wegen des Hemmungsapparates der glatten Muskelfasern in der Brustwarze keinen Milchfluss, bei gefüllten aber so lange Milchfluss bewirkt, bis Blutdruck, Milchdruck und Druck der Warzenmuskulatur auf die Enden der Milchgänge ins Gleichgewicht gekommen sind."

Henle ${ }^{3}$ ) sagt: „Man pflegt das Ausfliessen der Milch aus dem plötzlichen Zuströmen der Milch, d. h. aus einer raschen congestiven Dresden.

1) Berichte und Studien aus der Königl. sächsischen Entbindungsanstalt

2) Dieses Archiv, Bd. VII.

3) Handbuch der Eingeweidelehre, Bd. II. 
Vermehrung der Secretion zu erklären. Es verdient aber die Frage erwogen zu werden, ob nicht eine plötzliche Verminderung des den Ausfluss hemmenden Widerstandes an dieser Erscheinung betheiligt ist. Wenn man zwischen den mütterlichen Empfindungen und den Muskeln der Warze eine ähnliche Beziehung annimmt, wie zwischen erotischen Vorstellungen und den Muskeln der cavernösen Körper, so liesse sich begreifen, warum das Geschäft des Saugens mit einer Erschlaffung der Warzenmuskulatur beginnt, mit welchem zugleich eine Vergrösserung der Warze und eine Vermehrung ihres Blutreichthums verbunden wäre."

Diese Theorie Henle's hat Kehrer durch seine Beobachtungen an Milchfisteln widerlegt, bei denen während der Ruhe nichts, beim Einschiessen Milch aus Warze und Fistel zugleich floss, was nach der Henle'schen Theorie physikalisch nicht zu erklären ist.

Die Thatsache, die auch Kehrer kennt, dass ,auch bei reichlich secernirenden Frauen schwacher und kein Fluss vorkommen kann", lässt sich durch die Kehrer'sche Theorie nicht erklären.

Warum soll bei diesen die Hyperämie geringer sein? Schwergehende Warzen allen diesen Frauen zu imputiren, geht ebenfalls nicht an - unter den 37 Fällen habe ich bei einem den Eindruck gehabt, dass dieser Zustand vorliegt.

Nehmen wir dazu noch die Beobachtung, dass der Spontanfluss so häufig auftritt und oft ohne jedes subjectives Empfinden der Frau, so glaube ich nicht fehlzugehen, ein wesentliches Moment der Thätigkeit der Warzen-Muskulatur für das Ausbleiben, resp. Zustandekommen des Milchflusses zuzuschreiben. ${ }^{1}$ )

Während die Muskulatur bei den Frauen ohne Milchfluss dem Inhaltsdruck gewachsen ist, so wird bei den anderen die Grenze ihrer Leistungsfähigkeit oft schon durch den Druck der producirten Milch allein - Spontanfluss, sonst durch die durch Saugen reflectorisch angeregte Hyperämie - Saugfluss überschritten.

Die Ausflussmenge wird demnach von dem Grade der Schwäche der Muskulatur, der Milchproduction und Hyperämie der Drüsen abhängen.

Von der Kraft des Druckes, der beim Ausfliessen zur Anwendung kommt, konnte ich mich bei einigen Versuchen der Unter-

1) Auch Herff nimmt bei seinem Fall eine Erschlaffung der Muskulatur als wesentlich an. 
drückung des Saugflusses durch manuelle Compression der Warze, wie es Kehrer empfiehlt, überzeugen. Der Druck auf die Warze musste ein paar Mal recht kräftig sein und sofort nach dem Nachlassen floss Milch zwischen den Fingern hindurch (XV., XVII.).

Wurde während der ganzen Zeit des Stillens comprimirt, so lief unmittelbar darnach eine grössere oder kleinere Menge aus (XVIII., XX.).

Die Beobachtung, dass bei I- und II parae Milchfluss viel häufiger als bei Mehrgebärenden ist, lässt sich vielleicht durch einen Vorgang gleich der Arbeitshypertrophie der Muskeln erklären, durch das vorhergegangene Sauggeschäft bei der Warzenmuskulatur bedingt.

Pathologisch-anatomische Veränderungen habe ich mit Ausnahme des Falles XXV. nicht wahrnehmen können; die von Prochownik ${ }^{1}$ in 2 Fällen beschriebene Erweiterung der Milchgänge habe ich nie gesehen.

Fall XXV. hatte ein Lipoma mammae links. Dass dasselbe von keinem Einflusse war, zeigen die von beiden Brüsten annähernd gleich gelieferten Milchmengen.

Prochownik und Herff empfehlen gegen den Milchfluss die Elektricität, in der That ein Mittel, von dem man eine günstige Einwirkung auf die Muskulatur erwarten kann.

Bei nur 11 Beobachtungstagen lässt sich ein bestimmtes Urtheil nicht gewinnen; ich beschränke mich darauf, in Kurzem die gemachten Erfahrungen aufzuzählen. Es wurden jeweils, sowohl in dieser Beobachtungsweise, als auch in früheren Fällen, mit schwachem inducirten Strom die Warzenhöfe beiderseits je 5 bis 10 Minuten zweimal täglich elektrisirt.

Fall XIII. starker Spontanfluss; gleich nach dem ersten Mal Elektrisiren laufen sofort nachher 25,2 ab. Den Tag darauf kein Spontanfluss nach dem Elektrisiren, Saugfiuss geringer. Am 3. Tage fast kein Spontanfluss mehr, nach dem Elektrisiren läuft wenig aus. Der Ausfluss in der Nacht bleibt gleich.

Fall XV., XVII. kein Einfluss zu bemerken.

Fall XXIII. Während am 1. Tage nach dem Elektrisiren Milchfluss nicht eingetreten, fand dies nach dem Elektrisiren am 2. Tage wieder statt.

Fall XXV. Elektricität machtlos, sofort nachher wieder Fluss.

1) Centralblatt für Gynäkologie $1878, \mathrm{Bd}$. I. 
In manchen früheren Fällen soll nach Angabe von Prof. Fehling durch Anwendung des inducirten Stromes ein guter Erfolg beim Milchfluss erzielt worden sein.

Betonen wir nochmals, dass die durch Saug- und Spontanfluss verloren gehende Milch meist zwischen 8-18 Proc. der vom Säugling getrunkenen Milch beträgt, so liegt die praktische Bedeutung dieser Störung auf der Hand.

Bei der noch nicht zur Entscheidung gebrachten Frage über Einfluss der Elektricität auf die Milchbildung überhaupt und die Verhinderung des Abflusses und bei der Unsicherheit, durch Darreichung von Arzneimitteln diese Störung zu bekämpfen, halte ich es für das Rationellste, die Milch mit einem Milchfänger aufzufangen und so dem Säugling zu erhalten, was um so weniger versäumt werden sollte im Hinblick auf die Thatsache, dass der Milchfluss kein Zeichen für genügenden Milchvorrath ist. Die Sterilisirung der so aufgefangenen Muttermilch dürfte ja keine zu grossen Schwierigkeiten machen.

Es erübrigt noch, einige Versuche mitzutheilen, die auf der hiesigen Klinik mit Antipyrin und Extractum Galegae angestellt worden sind.

Guibert ${ }^{1}$ ) empfiehlt zur Unterdrückung der Milchsecretion Antipyrin täglich $2 \mathrm{~g}$, in Dosen von 0,25 .

I. Fall. Geburt an 18. August, hat gestillt bis 16. October; hatte Parametritis, Reconvalescent; da Patientin nach Entlassung aus der Anstalt ihr Kind nicht weiter stillen kann, wird dasselbe abgesetzt. 17., 18., 19. $8 \mathrm{Mal} \mathrm{0,25} \mathrm{Antipyrin} \mathrm{täglich,}$

19. $6 \mathrm{Uhr}$ Nachm. Milch reichlich ausdrüickbar.

II. Fall. Geburt am 12. October, Kind faultodt.

16., 17., 18., 19, 20. Milch noch reichlich vorhanden.

III. Fall. Geburt am 21. October, Kind todtgeboren.

24. starke Spannung der Brüste,

24., 25., 26. Antipyrin,

27. Antipyrin weggelassen ohne Erfolg.

IV. Fall. Geburt am 30. September, Mastitis sinistra, Incision.

19. October: Brüste sehr gespannt,

20. " hat aufgehört zu Stillen,

22., 23., 24. Antipyrin,

24. Abends Stat, idem.

V. Fall, Geburt am 16. October, Mastitis rechts, stillt links weiter. 22., 23., 24., 25., 26. October Antipyrin, 26. October Brüste noch voll Milch.

1) Archives de Tocologie et de Gynécologie 1891. 
VI. Fall, Geburt am 19. Januar, Abort mensis V.

21. Januar Briiste gespannt,

21., 22., 23. Januar Antipyrin,

24. Januar Brüste weniger gespannt. Aussetzen von Antipyrin,

26. Januar Brüste nicht mebr gespannt.

Bei Fall XV. hörte der Milchfluss bei Gabe von 2 g Antipyrin auf; bei einem anderen Falle aber, der nicht unter den beobachteten 25 Fällen ist, und bei dem täglich ca. $200 \mathrm{ccm}$ theils spontan, theils beim Stillen auslief, hatte es keine Wirkung.

24. Januar 7 Mal 0,25 den Tag über laufen $220 \mathrm{~g}$ aus,

25. $" 2,0,25$ bis Mittag ca. $120 \mathrm{~g}$.

Dass bei Fall XV. der Zufall mitgespielt, ist mir nach den sonstigen Erfahrungen mit Antipyrin sehr wahrscheinlich.

Gu ibert hat das Antipyrin meist 6-8 Tage gegeben, eine Zeit, in der erfahrungsgemäss die Milchstauung von selbst zurückgeht.

Das in den Therapeutischen Monatsheften als Mittel zur Erhöhung der Milcbabsonderung empfohlene Extractum Galegae ist in der Dosis :

Extr. Galeg. 10,0

Aq. Mentb. 40,0

3. Mal täglich einen Kaffeelöffel.

in 8 Fällen ohne jeglichen Erfolg gegeben worden.

Wir sind daher nach den Erfahrungen auf der hiesigen Klinik nicht im Stande, die milchhemmende Wirkung des Antipyrin, die milchtreibende der Galega anzuerkennen. 